\title{
RECTILINEAR AND BROWNIAN MOTION FROM A RANDOM POINT IN A CONVEX REGION
}

\author{
PETER EHLERs ${ }^{1}$, ERNEST ENNS $^{2}$ AND TAK FunG ${ }^{3}$ \\ ${ }^{1}$ Department of Mathematics and Statistics, University of Calgary, Calgary, Alberta, Canada, ${ }^{2}$ Department of \\ Mathematics and Statistics, University of Calgary, Calgary, Alberta, Canada, ${ }^{3}$ Information Technologies, \\ University of Calgary, Calgary, Alberta, Canada \\ e-mail: ehlers@math.ucalgary.ca \\ (Accepted June 7, 2001)
}

\begin{abstract}
A particle is projected from a point $P$ in a subset $E$ of a convex region $H$ to a point $Q$ in a uniformly random direction. The probability that $Q$ lies in the interior of $H$ at time $t$ is obtained for two types of motion of the particle, rectilinear (i.e. straight-line) and Brownian. In the case of rectilinear motion, the first passage time through the boundary of $H$ is considered. Results are obtained in terms of the generalized overlap function for embedded bodies.
\end{abstract}

Keywords: Brownian motion, convex body, geometric probability, random ray.

\section{INTRODUCTION}

Consider a particle moving from point $P$ at time $t=0$ to point $Q$ at $t>0 . P$ is taken to lie in a subset $E$ of a compact convex body $H \subset R^{n}$. The set $E$ may be nonconvex. It may be disconnected and may have lower dimensionality than $H$; indeed, $E$ may consist of a single point (see Fig. 1). In the next section we relate the probability that $Q$ lies in the interior of $H$ to the generalized overlap function for embedded bodies introduced by Enns and Ehlers (1988). Thereafter we obtain this probability first for the case of motion along a straight path and then for the case of Brownian motion. Depending on its equations of motion, the particle may leave and re-enter the region $H$ any number of times. We are concerned only with its presence or absence inside $H$ at time $t$.

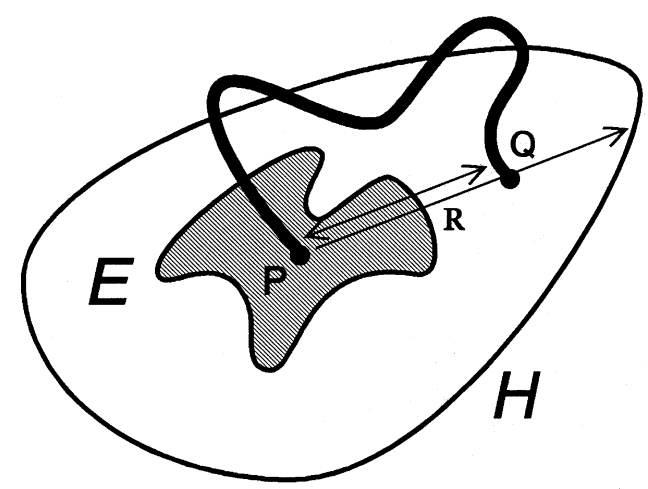

Fig. 1. A particle moves from $P$ to $Q$ in time $t$.
There is a considerable literature on escape processes and first exit times, especially as related to the example of Brownian motion; see, for example, Getoor (1979) or Wendel (1980). For more general books, see Gihman and Skorohod (1975) or Knight (1981).

\section{GENERAL FORMULATION}

Let $Q(t)$ denote the location of the particle at time $t \geq 0$. Let $P=Q(0)$ be a uniformly random starting point in $E$. Denote the distance between $Q(0)$ and $Q(t)$ by $X(t)$. Assuming that motion is isotropic, it is possible to relate the probability of finding $Q(t)$ inside $H$ to the overlap function which is defined by

$$
\Omega_{E, H}(x) \equiv \frac{E_{\theta}[\operatorname{vol}(E(x, \theta) \cap H]}{\operatorname{vol}(E)}
$$

where $E(x, \theta)$ is the translate of $E$ in direction $\theta$ by a distance $x, \operatorname{vol}(\bullet)$ denotes volume and $\mathbf{E}_{\theta}(\bullet)$ indicates direction average with $\theta$ uniformly random over all directions.

Let

$$
h(t)=\mathbf{P}(Q(t) \in H)
$$

and 


$$
F_{X}(r, t)=\mathbf{P}(X(t) \leq r) .
$$

The following theorem relates $h(t)$ to the overlap function.

Theorem

$$
h(t)=\int \Omega_{E, H}(r) d F_{X}(r, t) .
$$

Proof. Let $B(r, P)$ denote the $n$-ball of radius $r$ with centre at $P$ and let $\partial B(r, P)$ denote its surface. Then

$$
\begin{aligned}
\mathbf{P}(Q(t) \in H \mid X(t)=r ; Q(0)=P) & =\frac{\operatorname{vol}[H \cap \partial B(r, P)]}{\operatorname{vol}[\partial B(r, P)]} \\
& =\frac{\operatorname{vol}[H \cap \partial B(r, P)]}{n C_{n} r^{n-1}} \\
& =\frac{\phi(r, P)}{n C_{n}}
\end{aligned}
$$

where $C_{n}=\pi^{n / 2} / \Gamma\left(\frac{n}{2}+1\right)$ is the volume of the unit $n$-ball and $\phi(r, P)$ is the solid angle subtended at $P$ by $H \cap \partial B(r, P)$. Averaging with respect to net distance $X$ and initial point $P$, one obtains the unconditional probability that $Q(t) \in H$ :

$$
\begin{aligned}
h(t) & =\mathbf{P}(Q(t) \in H) \\
& =\mathbf{E}_{P} \mathbf{E}_{X} \mathbf{P}(Q(t) \in H \mid X(t) ; P) \\
& =\int_{r=0}^{\infty} \mathbf{E}_{P}\left[\frac{\phi(r, P)}{n C_{n}}\right] d F_{X}(r) .
\end{aligned}
$$

But $\mathbf{E}_{P}\left(\phi(r, P) / n C_{n}\right)=\Omega_{E, H}(r) \quad($ Enns and Ehlers, 1988). Therefore

$$
h(t)=\int \Omega_{E, H}(r) d F_{X}(r, t)=\mathbf{E}_{X}\left[\Omega_{E, H}(X(t))\right],
$$

relating $h(t)$ and the overlap function.

It was shown by Enns and Ehlers (1988) that $1-\Omega_{E, H}(r)$ is the distribution function of the length $R$ of a random ray generated by selecting a point in $E$ and a direction, independently uniformly distributed and with terminal point in the boundary $\partial H$ of $H$. We call this a $v$-random ray. Writing $\Omega_{E, H}(r)=\mathbf{P}(R>r)=\bar{F}_{R}(r)$ results in

$$
\begin{aligned}
h(t) & =\int \bar{F}_{R}(r) d F_{X}(r, t) \\
& =\int F_{X}(r, t) d F_{R}(r) \\
& =\mathbf{E}_{R}\left(F_{X}(R, t)\right) .
\end{aligned}
$$

Summarizing, we have

$$
h(t)=\mathbf{E}_{X}\left[\Omega_{E, H}(X(t))\right]=\mathbf{E}_{R}\left(F_{X}(R, t)\right),
$$

where the $R$-expectation is with respect to $v$ measure.

We now turn to specific types of particle motion.

\section{RECTILINEAR MOTION}

For a particle undergoing straight-line motion with position function $u(t)$ along the directed halfline originating at $P$ (direction equal to the direction of motion so that $u(t)$ is increasing), $X(t)$ has degenerate distribution

$$
F_{X}(r, t)=I(r-u(t)),
$$

where $I(\bullet)$ is the indicator function

$$
I(x)= \begin{cases}1 & \text { if } x>0 \\ 0 & \text { if } x \leq 0 .\end{cases}
$$

Substitution in Eq. 2 yields

$$
h(t)=\Omega_{E, H}(u(t)) .
$$

With $\partial H$ denoting the boundary of $H$, we define the random variable

$$
T=\min \{t: Q(t) \in \partial H\} .
$$

Thus $T$ is the first exit time for a particle. It follows that $h(t)=\mathbf{P}(T>t) \equiv \bar{F}_{T}(t)$. Moments of $T$ may then be obtained by evaluating

$$
\mathbf{E} T^{k}=k \int_{t=0}^{\infty} t^{k-1} \bar{F}_{T}(t) d t=k \int_{t=0}^{\infty} t^{k-1} \Omega_{E, H}(u(t)) d t .(3)
$$

For the case of motion at constant velocity, where $u(t)=v t$, Eq. 3 yields

$$
\mathbf{E} T^{k}=v^{-k} k \int_{x=0}^{\infty} x^{k-1} \Omega_{E, H}(x) d x .
$$

Obviously, this case corresponds to $T=R / v$, where $R$ is the $v$-random ray length from a uniformly random point in $E$ to the boundary of $H$. A shape-independent moment for $n$-dimensional $H$ is

$$
\mathbf{E} T^{n}=\frac{\operatorname{vol}(H)}{C_{n} v^{n}} .
$$

Example. Enns and Ehlers $(1988,1993)$ give the overlap functions for the case of concentric balls. Let $H=B_{n}(b), E=B_{d}(a), a \leq b$, and $d \leq n=3$. 
This situation models the physically important cases of particles generated in a linear or circular region inside the 3-ball. We have $\Omega_{E, H}(x)=1$ for $x \leq b-a$ and $\Omega_{E, H}(x)=0$ for $x \geq b+a$. In the interval $b-a<x<b+a$, the overlap functions for $d=1,2,3$ are:

$d=1$ :

$\Omega_{E, H}(x)=\frac{1}{8 a x}\left[\left(b^{2}-a^{2}\right)+4 a x+2 b x-3 x^{2}+2\left(x^{2}-b^{2}\right) \log \left|\frac{x-b}{a}\right|\right]$

$d=2$ :

$\Omega_{E, H}(x)=\frac{1}{6 a^{2} x}\left[3 a\left(b^{2}-a^{2}-x^{2}\right)+3 x\left(a^{2}+\left|x^{2}-b^{2}\right|\right)+2\left(a^{3}-\left|x^{3}-b^{3}\right|\right)\right]$

$d=3$ :

$\Omega_{E, H}(x)=\frac{1}{16 a^{3} x}\left[-3\left(b^{2}-a^{2}\right)^{2}+8\left(a^{3}+b^{3}\right) x-6\left(a^{2}+b^{2}\right) x^{2}+x^{4}\right]$

The integrations for the moments are elementary. In each case, the moments may be written in the form

$$
\mathbf{E} T^{k}=\left(\frac{b}{v}\right)^{k}\left(m_{d}(k, \lambda)+m_{d}(k,-\lambda)\right)
$$

where $\lambda=a / b$. The functions $m_{d}(k, \lambda)$ are listed in the appendix. The first and second moments for all three cases are:

$d=1$ :

$\mathbf{E} T=\frac{b}{v}\left[\frac{1}{4}+\frac{1-\lambda^{2}}{8 \lambda} \log \left(\frac{1+\lambda}{1-\lambda}\right)+\frac{1}{2} \int_{0}^{1} \frac{\log x}{\lambda^{2} x^{2}-1} d x\right]$

$\mathbf{E} T^{2}=\frac{b^{2}}{v^{2}}\left(1-\frac{\lambda^{2}}{9}\right)$

$d=2$ :

$\mathbf{E} T=\frac{b}{v}\left[\frac{1}{3}+\frac{\log \left(1-\lambda^{2}\right)}{3 \lambda^{2}}+\frac{3-\lambda^{2}}{6 \lambda} \log \left(\frac{1+\lambda}{1-\lambda}\right)\right]$

$\mathbf{E} T^{2}=\frac{b^{2}}{v^{2}}\left(1-\frac{\lambda^{2}}{6}\right)$

$d=3$ :

$\mathbf{E} T=\frac{b}{v}\left[\frac{3\left(1+\lambda^{2}\right)}{8 \lambda^{2}}-\frac{3\left(1-\lambda^{2}\right)^{2}}{16 \lambda^{3}} \log \left(\frac{1+\lambda}{1-\lambda}\right)\right]$

$\mathbf{E} T^{2}=\frac{b^{2}}{v^{2}}\left(1-\frac{\lambda^{2}}{5}\right)$

Clearly, more complicated position functions $u(t)$ may be substituted in Eq. 3, leading to relatively straight-forward tedious integrations. Note that, depending on the form of $u(t)$, a particle may leave and re-enter $H$ in $(0, t)$.

One modification of practical interest for constant-speed motion is the case of radioactive particles with short lifetimes. Such particles may decay before reaching the boundary of $H$. Let $T_{d}$ denote the random lifetime of the particle (time to decay, given birth at point $P$ ) and let the random time to reach the boundary in the absence of decay be $T_{0}$. If the decay process is independent of the particle's motion, then the particle vanishes unless $t<\min \left(T_{0}, T_{d}\right)$. Then

$$
\begin{aligned}
h(t) & =\mathbf{P}\left(T_{0}>t\right) \mathbf{P}\left(T_{d}>t\right) \\
& =\bar{F}_{T_{0}}(t) \bar{F}_{T_{d}}(t) \\
& =\Omega_{E, H}(v t) \bar{F}_{T_{d}}(t) .
\end{aligned}
$$

If the particles have fixed lifetime $\tau$, then $T_{d}$ has degenerate distribution and

$$
h(t)= \begin{cases}\Omega_{E, H}(v t) & \text { if } t<\tau \\ 0 & \text { if } t \geq \tau\end{cases}
$$

\section{BROWNIAN MOTION}

For particles undergoing Brownian motion (with diffusion constant $D)$ the net distance $X(t)$ has probability density function

$$
f_{X}(r, t)=\frac{n C_{n} r^{n-1} \exp \left(-r^{2} / 4 D t\right)}{(4 \pi D t)^{n / 2}} .
$$


One application is the situation where an experimenter can make observations only in a limited field of view (under a microscope, say). An identifiable cell might be observed to be in a certain part of the field of view at time zero and then might be observed again later.

If we expand $F_{X}(R, t)$ as used in Eq. 2 in a Taylor series in $R$, we find

$$
\begin{aligned}
h(t) & =\mathbf{E}_{R}\left[\sum_{k=0}^{\infty} F_{X}^{(k)}(0, t) \frac{R^{k}}{k !}\right] \\
& =\mathbf{E}_{R}\left[\sum_{k=0}^{\infty} f_{X}^{(k-1)}(0, t) \frac{R^{k}}{k !}\right] \\
& =\sum_{k=0}^{\infty} f_{X}^{(k-1)}(0, t) \frac{\mathbf{E}_{R} R^{k}}{k !}
\end{aligned}
$$

which gives $h(t)$ in terms of moments of the length of a $v$-random ray.

Remark. It seems physically curious that, since $f_{X}^{(k-1)}(0, t)=0$ for $k<n, h(t)$ depends only on

moments of $R$ that are of dimensional order or greater.

If we consider again concentric balls where $E=B_{d}(a)$ and $H=B_{n}(b)$, with $d \leq n \leq 3$ and $a \leq b$, then the results of Enns and Ehlers (1993) may be used to evaluate $h(t)$. The expressions are, in general, complicated but not difficult to derive. For the case of $a=b$ and $d=n$ we obtain for $n=2$ :

$$
h(t)=\frac{1}{\sqrt{\pi}} \sum_{k=1}^{\infty} \frac{(-1)^{k+1} \Gamma\left(k+\frac{1}{2}\right)}{k !(k+1) !}\left(\frac{b^{2}}{D t}\right)^{k}
$$

and for $n=3$ :

$$
h(t)=\frac{3}{\sqrt{\pi}} \sum_{k=1}^{\infty} \frac{(-1)^{k+1}}{(2 k+1)(k+1)(k+2)(k-1) !}\left(\frac{b^{2}}{D t}\right)^{k+\frac{1}{2}} .
$$

Fig. 2 shows how $h(t)$ decreases with time.

For the case of $a<b$ and $d=n=3$ we find

$$
h(t)=q(t ; a, b)+q(t ;-a, b)
$$

where

$$
\begin{aligned}
q(t ; a, b)= & \frac{\exp \left(-(b+a)^{2} / 4 D t\right)}{4 \sqrt{\pi} a^{3}}\left[2\left(a^{2}+b^{2}-a b\right) \sqrt{4 D t}-(4 D t)^{3 / 2}\right] \\
& +\left(1+\frac{b^{3}}{a^{3}}\right)\left[\Phi\left(\sqrt{2} \frac{b+a}{\sqrt{4 D t}}\right)-\frac{1}{2}\right]
\end{aligned}
$$

Here $\Phi(\bullet)$ is the cumulative standard normal distribution function. Fig. 3 shows $h(t)$ for selected values of $a$ with $b=1$.

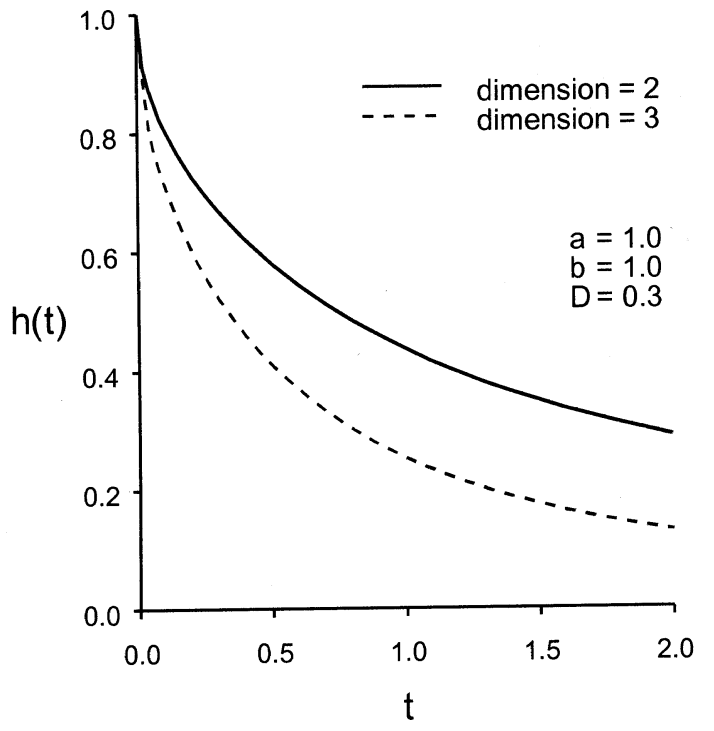

Fig. 2. The probability $h(t)$ for circular or spherical regions: $E=H$.

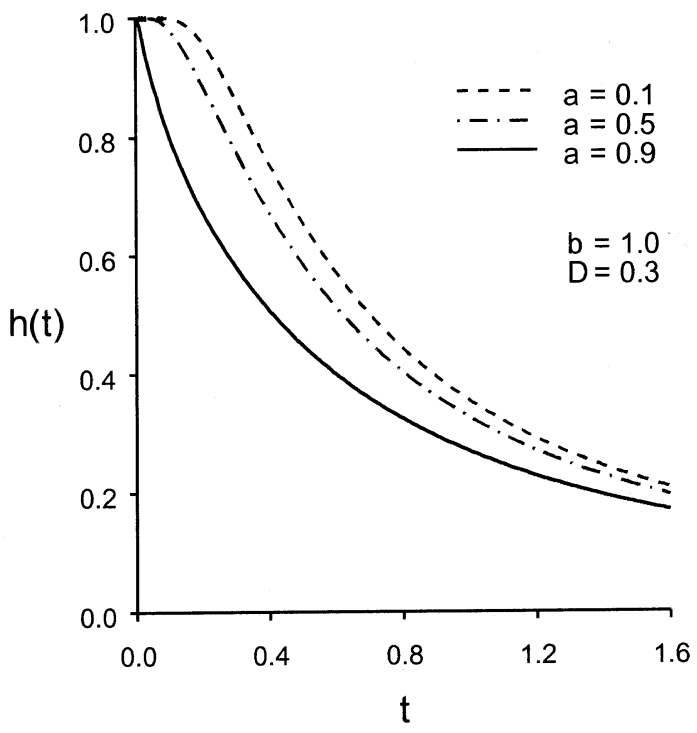

Fig. 3. The probability $h(t)$ for concentric spherical regions $E$ and $H$. 


\section{APPENDIX}

$$
\begin{aligned}
& m_{1}(k, \lambda)=\frac{(1+\lambda)^{k}(1-k \lambda)}{4 k\left(k^{2}-1\right) \lambda}+\frac{1}{4} \sum_{j=0}^{\infty} \frac{\lambda^{2 j}}{(2 j+1)^{2}}\left[\left(\begin{array}{c}
k \\
2 j
\end{array}\right)+\left(\begin{array}{c}
k-2 \\
2 j
\end{array}\right)\right] \\
& m_{2}(k, \lambda)=\frac{1}{3 \lambda^{2}}\left(\frac{1}{k+2}+\frac{1}{k-1}\right)-\frac{\left(2-3 \lambda+\lambda^{3}\right)(1+\lambda)^{k-1}}{6 \lambda^{2}(k-1)}+\frac{(1+\lambda)^{k+1}}{2 \lambda(k+1)}-\frac{(1+\lambda)^{k+2}}{3 \lambda^{2}(k+2)} \\
& m_{3}(k, \lambda)=\frac{3(1+\lambda)^{k+1}}{2(k+3)\left(k^{2}-1\right)}\left(\frac{k+1}{\lambda^{2}}-\frac{1+\lambda^{2}}{\lambda^{3}}\right)
\end{aligned}
$$

\section{REFERENCES}

Enns EG, Ehlers PF (1988). Chords through a convex body generated from within an embedded body. J Appl Probab 25:700-7.

Enns EG, Ehlers PF (1993). Notes on random chords in convex bodies. J Appl Probab 30:889-97.

Getoor RK (1979). The Brownian escape process. Ann Probab 7:864-7.
Gihman IL, Skorohod AV (1975). The Theory of Stochastic Processes II. Springer-Verlag, New York.

Knight FB (1981). Brownian Motion and Diffusion. American Mathematical Society, Mathematical Surveys 18.

Wendel JG (1980). Hitting spheres with Brownian motion. Ann Probab 8:164-9. 